\title{
Origin of the Galactic Diffuse X-ray Emission: Fe Line Diagnostics with Suzaku
}

\section{Masayoshi Nobukawa*}

Faculty of Education, Nara University of Education, Takabatake-cho, Nara, Nara 630-8528, Japan

E-mail: nobukawa@nara-edu.ac.jp

\section{Kumiko K. Nobukawa}

Department of Physics, Faculty of Science, Nara Women's University, Kitauoyanishi-machi, Nara, Nara 630-8506, Japan

Email: kumiko@cc.nara-wu.ac.jp

\section{Shigeo Yamauchi}

Department of Physics, Faculty of Science, Nara Women's University, Kitauoyanishi-machi, Nara, Nara 630-8506, Japan

\section{Hideki Uchiyama}

Faculty of Education, Shizuoka University, 836 Ohya, Suruga-ku, Shizuoka, Shizuoka 422-8529, Japan

\section{Katsuji Koyama}

Department of Physics, Graduate School of Science, Kyoto University, Kitashirakawa-oiwake-cho, Sakyo-ku, Kyoto, Kyoto 606-8502, Japan

\begin{abstract}
We investigated the origin of the Galactic diffuse X-ray emission (GDXE) using the archive data taken by Suzaku focusing on the Fe lines, Fe I K $\alpha, \mathrm{Fe}$ XXV He $\alpha$, and Fe XXVI Ly $\alpha$, separately. From the detailed flux profile of $\mathrm{Fe} \mathrm{XXV} \mathrm{He} \alpha$, we decomposed the GDXE into three components: Center, Ridge, and Bulge. Since the Fe I K $\alpha$ and Fe XXV He $\alpha$ lines have different spatial distributions, they should have different origins. We also modeled Fe-band spectra of X-ray active stars (XAS), magnetic cataclysmic variables (mCV), non-magnetic cataclysmic variables (non$\mathrm{mCV}$ ), and active binaries (AB) using the Suzaku archive data. The X-ray spectrum near the Fe $\mathrm{K}$-shell lines of Bulge is well explained by the non-mCVs-dominant plasmas with a small contribution of mCVs and ABs. The Center and Ridge spectra still remain significant residuals with any combination of the point sources.
\end{abstract}

XII Multifrequency Behaviour of High Energy Cosmic Sources Workshop

\section{2-17 June}

Palermo, Italy

\footnotetext{
* Speaker.
} 


\section{Introduction}

There is diffuse $\mathrm{X}$-ray emission that are not resolved into bright $\mathrm{X}$-ray sources along the Galactic plane, which is called the Galactic diffuse X-ray emission (GDXE). The origin has been discussed for a long time since its discovery [1]. One of the major scenarios is integration of faint point sources, which cannot be resolved due to instrumental spacial resolution [2, 3]. Cataclysmic variables $(\mathrm{CVs})$ and/or active binaries (ABs) are thought to be possible candidates. Another scenario is truly diffuse hot plasma with high temperature $\sim 10 \mathrm{keV}$ [4]. If this is the case, the total thermal energy is estimated to be $\sim 10^{54} \mathrm{erg}$, which corresponds to $\sim 1000$ supernovae. The high temperature plasma cannot be bound by gravitational potential of the Galaxy, and should escape in $10^{5}$ years. Huge energy injection corresponding to one supernova per 100 years is required.

The characteristic feature of the GDXE is K-shell emission lines of Fe at $6.40 \mathrm{keV}$ (neutral $\mathrm{Fe}, \mathrm{Fe}$ I $\mathrm{K} \alpha$ ), $6.68 \mathrm{keV}$ (He-like Fe, Fe Xxv He $\alpha$ ), and $6.97 \mathrm{keV}$ (H-like Fe, Fe Xxvi Ly $\alpha$ ), which were discovered by the X-ray CCD camera onboard ASCA [5]. The Fe I $\mathrm{K} \alpha$ line generated in cold material which is irradiated by high energy particles (X-rays or cosmic rays). On the other hand, the Fe XXv $\mathrm{He} \alpha$ and Fe XXvi Ly $\alpha$ lines come from high temperature plasma.

Some previous studies focused on the Fe lines in order to investigate the GDXE origin. RXTE/PCA observation found that the intensity profile of the Fe line along the Galactic longitude and latitude resembles that of near infrared stars [6]. This fact supports the point-source scenario. However, the instrument has a limited spacial resolution less than the height of the latitudinal distribution $\sim 1^{\circ}$, and furthermore it has too limited energy resolution to separate the three Fe K-shell lines.

Chandra performed $1 \mathrm{Msec}$ observation toward the southern region of the Galactic center, at $(l . b)=\left(0 .{ }^{\circ} 0,1 .{ }^{\circ} 4\right)[3]$. An important result was obtained that more than $80 \%$ of the X-ray emission at $\mathrm{Fe}$ line band was resolved into discrete sources. It appears strong evidence that suggests the point-source scenario. In that observation, however, the Fe lines were not separated. We should also note that the observation was limited to a small area (a circle of radius $\sim 2^{\prime}$ ).

The X-ray Imaging Spectrometers (XIS: [7]) onboard Suzaku has an advantage in the GDXE observation; the instrumental background for diffuse emission is the lowest and most stable, and the sensitivity and the energy resolution in the Fe line band is the highest among the current or past X-ray observatories. By Suzaku observation, [8] separated the GDXE into three components: the Galactic center (GCXE), Galactic bulge (GBXE), and Galactic ridge (GRXE) X-ray emissions. They also performed spectral analysis and found that the GCXE and GRXE have different spectra to each other; the plasma temperature varies by location.

Analyzing the Suzaku archive data from a large number of pointing positions along the Galactic plane, we measured the scale heights of the GCXE, GRXE, and GBXE, separately [9]. Furthermore, we determined accurate equivalent widths (EW) of the Fe I K $\alpha$ Fe XXv He $\alpha$ and Fe XxvI Ly $\alpha$ for each component of the GDXE, separately, and also obtained mean spectra of the point sources ( $\mathrm{CVs}$ and $\mathrm{ABs}$ ). Then we tried fitting the GDXE spectra with the point-source models focusing on the Fe lines [10]. Detailed analyses are written in $[9,10]$. In this proceedings, we report the points in the two papers, and discuss the origin of the GDXE. 


\section{Observations}

In this study, we used the Suzaku data set near the Galactic plane with the number of pointing positions of 143 and total net exposure time of $8300 \mathrm{ksec}$. In principle, we extracted photons from the entire region of the XIS field of view (FOV; the size of the FOV was 17.' $8 \times 17 .^{\prime} 8$ ). Discrete sources in the FOVs are excluded. Detailed observation logs is shown in Table 1 in [9]. Data reduction procedure are given in $[9,10]$.

\section{Latitudinal and Longitudinal Distribution}

We investigate the spacial distribution of the $\mathrm{Fe} \mathrm{Xxv} \operatorname{He} \alpha$ line along the Galactic latitude, in the central and plane parts. An X-ray spectrum in the 4-10 keV band was extracted from each FOV and subtracted the non X-ray background (NXB). We fitted the spectra with an absorbed bremsstrahlung and many Gaussians as well as the cosmic X-ray background (CXB) model [11]. The detailed fitting model is written in [9]. Then the fluxes of $\mathrm{Fe}$ I $\mathrm{K} \alpha, \mathrm{Fe} \mathrm{xxv} \mathrm{He} \alpha$, and Fe XXvi Ly $\alpha$ lines were obtained.

We grouped the results into four regions. (a) $|l|<0 .{ }^{\circ} 5$, (b) $l=358 .{ }^{\circ} 5$, (c) $l=356 .{ }^{\circ} 0-356 .{ }^{\circ} 4$, and (d) $|l|=10^{\circ}-30^{\circ}$. Figure 1 shows the latitudinal distribution of the $\mathrm{Fe} \mathrm{Xxv} \mathrm{He} \alpha$ line emission in the regions (a) and (d). The data in the region (a) could not be represented by one simple powerlaw model. We assumed that there are two components and fitted fitted the profiles of (a), (b), and (c) with a two exponential model as represented in the left panel of figure 1. One component is concentrated on the center region with the e-folding latitude scale of $0 .{ }^{\circ} 26$, and the other is more spread with the scale of $2 .^{\circ} 25$. The region (d), on the other hand, shows a single component, and we fitted the data with a one-exponential model (right panel in figure 1). The e-folding latitude scale is $1 .^{\circ}$. Thus, the GDXE should be have three components: center (GCXE), bulge (GBXE), and ridge (GRXE).

Figure 2 shows the schematic view of the three components of the GDXE. Assuming a distance of $8 \mathrm{kpc}$, the e-folding scale heights of the GCXE, GBXE, and GRXE determined from the Fe XXv He $\alpha$ line profile are 30 pc, 300 pc, and 140 pc, respectively. Here the statistical uncertainty is about $10 \%$. The small area where deep Chandra observation was performed [3] is contained within the GBXE area. The scale height of the Fe XXV He $\alpha$ line in the GRXE may be barely consistent with those of CVs and ABs (see [9] and references therein).

We also measured the e-folding scale height of the Fe I K $\alpha$ line. In the GCXE, the scale height of the $\mathrm{Fe}$ I $\mathrm{K} \alpha$ line $(30 \mathrm{pc}$ ) is similar to that of the $\mathrm{Fe} \mathrm{XXV} \mathrm{He} \alpha$ lines. On the other hand, in the GBXE and GRXE, the scale height of the Fe I K $\alpha$ line (160 pc and $70 \mathrm{pc}$ for the GBXE and GRXE, respectively) is about a half of that of the $\mathrm{Fe} \mathrm{XXV} \mathrm{He} \alpha$ line. Since the typical statistical uncertainty is 30\% and $20 \%$ for the GBXE and GRXE. respectively, the difference between the Fe I K $\alpha$ and Fe XXv He $\alpha$ lines in the GBXE is marginal, but the difference in the GRXE is significant. Furthermore, the scale height of the Fe I K $\alpha$ line in the GRXE is smaller than that of CVs and ABs; it is similar to that of molecular clouds [12,13] rather than point sources.

We made the longitudinal profiles of the $\mathrm{Fe}$ lines. Figure 3 shows those of $\mathrm{Fe} \mathrm{I} \alpha \alpha$ and $\mathrm{Fe} x \mathrm{xv} \operatorname{He} \alpha$ lines, respectively. In the $\mathrm{Fe} \mathrm{Xxv} \operatorname{He} \alpha$ line profile (the middle panel), we can see a smooth and consistent curve between the east and west data, whereas that is not the case for the 
Fe I $\mathrm{K} \alpha$ line profile (the upper panel). The bottom panel shows the flux ratio between the two iron lines. We can see large excesses of the $\mathrm{Fe}$ I $\mathrm{K} \alpha$ line relative to the $\mathrm{Fe}$ Xxv $\mathrm{He} \alpha$ line on the Galactic ridge $\left(l=1 .{ }^{\circ} 5-3 .{ }^{\circ} 5\right.$ and $\left.l=330^{\circ}-340^{\circ}\right)$. Combined with the scale heights, the Fe I $\mathrm{K} \alpha$ and $\mathrm{Fe}$ XXV He $\alpha$ lines in the GRXE should be of the different origin. As for the region at $l=1 .{ }^{\circ} 5-3 .{ }^{\circ} 5$, the detailed analysis is reported in [14].
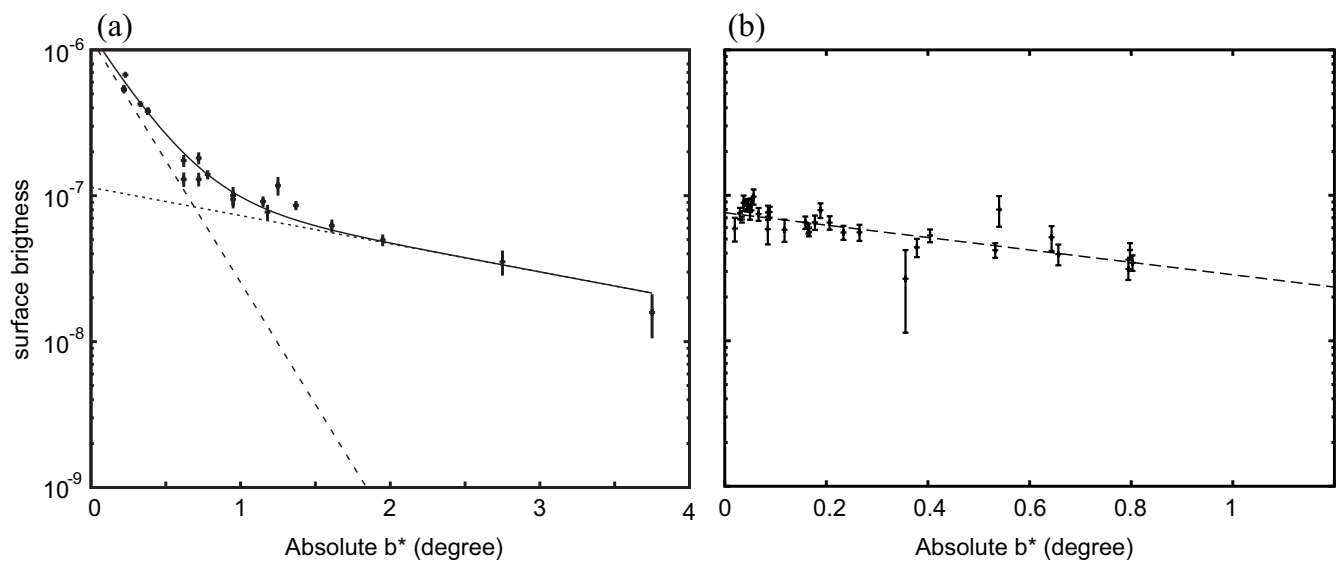

Figure 1: (a) Distribution of Fe XXV He $\alpha$ intensity along the Galactic latitude at $l \sim 0^{\circ}$. The solid line shows a two-exponential model with . (b) Same figure as (a) but at Ridge $\left(|l|=10^{\circ}-30^{\circ}\right)$.

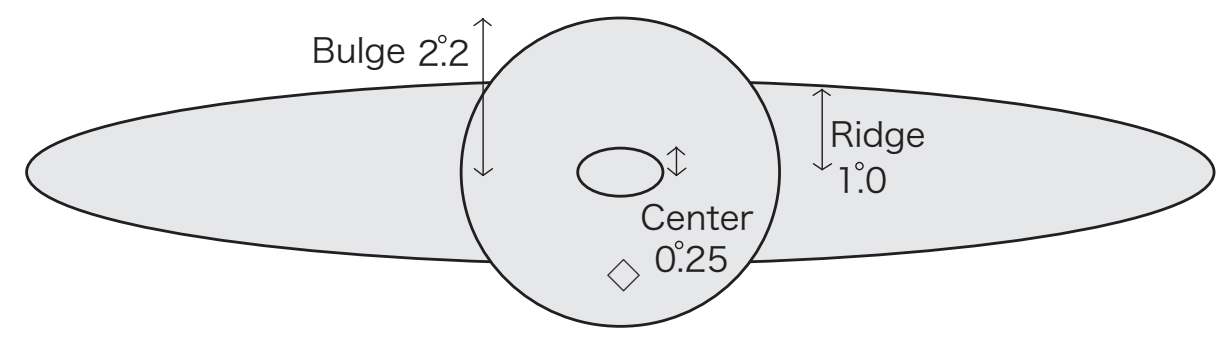

Figure 2: Schematic view of the GDXE in the Galaxy. The three components, "Center", "Bulge", and "Ridge" have scale heights $0^{\circ} .25,1^{\circ} .0$, and $2^{\circ} .2$, respectively, which are determined from the Fe XXV He $\alpha$ line intensity profiles along the Galactic latitude (Figure 1). The diamond mark represents the position of the Chandra deep observation [3].

\section{Fe Line Diagnostics}

We examined the Fe line spectra of the GDXE and the point sources. In the point source scenario, the candidate stars have been mainly, magnetic CVs (mCVs), non-magnetic CVs (non$\mathrm{mCVs}$ ), and $\mathrm{ABs}$ We extracted the mean spectra for the GDXE and individual classes of point sources and tried fitting the GDXE spectra with a combination of the point-source spectra. This method is applied partly by the previous work [15].

From the Suzaku archive data, we selected many point sources of mCVs, non-mCVs, and ABs: $26 \mathrm{mCV}$ s including symbiotic stars, intermediate polars, and polars, 13 non-mCVs, and $9 \mathrm{ABs}$. We analyzed each source, and fitted it with a thin-thermal plasma model to obtain the temperature, $\mathrm{Fe}$ 

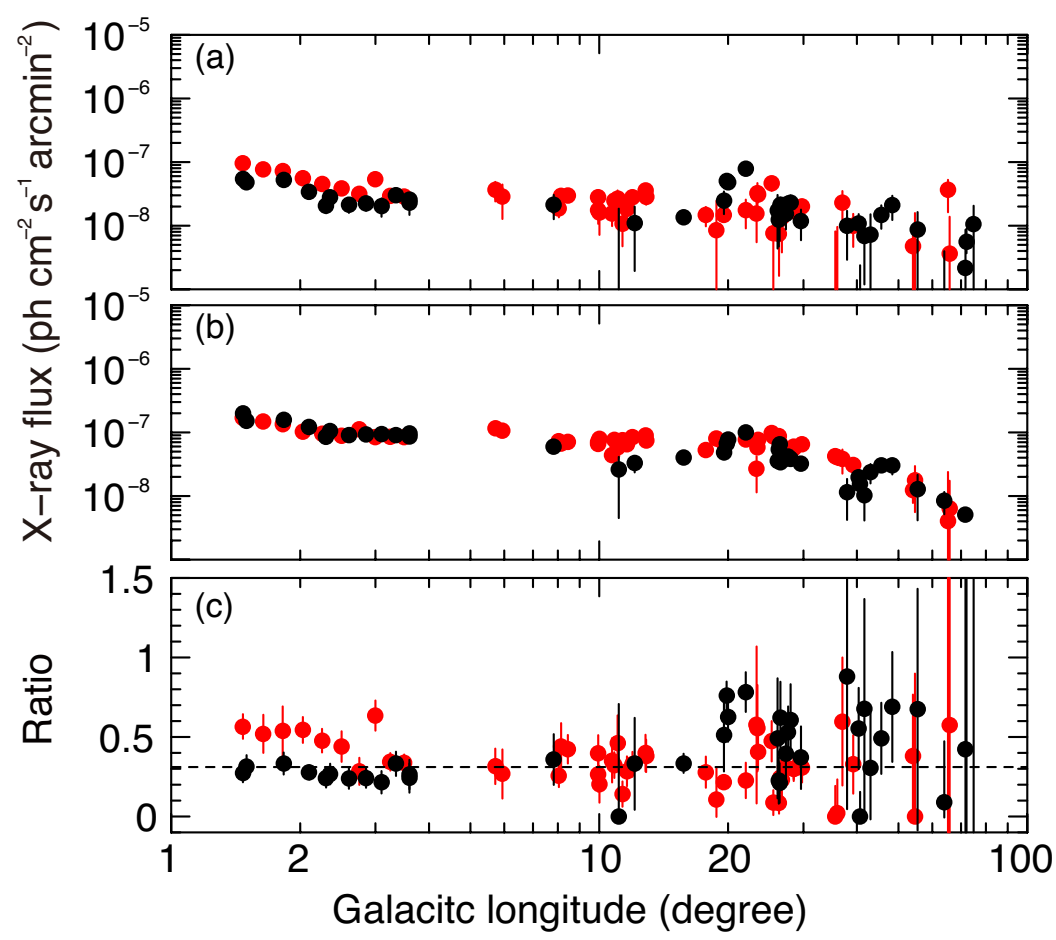

Figure 3: X-ray flux of Fe lines along the Galactic longitude. Black and red data are taken from the eastern $\left(l>0^{\circ}\right)$ and western $\left(l<0^{\circ}\right)$ sides, respectively. (a) For Fe I K $\alpha$. (b) For Fe XXV He $\alpha$. (c) Flux ratio of the Fe lines by dividing (a) by (b).

abundance, and luminosity. Figure 4 shows a temperature - luminosity plot of the mCVs, non$\mathrm{mCVs}$, and ABs. Data points distribute wide ranges of temperature and luminosity. Using the all the point sources in each class, we obtained the mean spectra. We should note that there are limited samples of point sources. In order to make a more correctly meaned spectrum of each class, we made modeling in each class with the parameters, such as abundance, temperature, EW of the $\mathrm{Fe}$ I $\mathrm{K} \alpha$ line, and X-ray luminosity function. Readers interested in the detailed procedure are referred to [10].

Then we try fitting the GDXE spectra, of the center, bulge, and ridge, with a combination of the point source spectra, focusing on the Fe line structures. Figure 5 shows the GBXE spectrum. Fitting the spectrum with a combination of the point source spectra results in a nice fit, in particular in the Fe line band. Thus the point-source model can explain the bulge spectrum, which is consistent with the result of Chandra observation contained within the GBXE area; $\sim 80 \%$ of the emission was resolved into point sources. Previous studies claimed that $\mathrm{mCV}$ are the dominant source of the GDXE $[2,3,16]$. In contrast, we found that the major fraction is occupied by non-mCVs, which is $\sim 70 \%$ of the total, and those of the mCVs and the ABs are less than $10 \%$ and $\sim 20-30 \%$, respectively.

We also applied this approach to the GRXE and GCXE. Figure 6 (a) shows the result of the ridge. We could not obtained good fitting; there are still large residuals at the $\mathrm{Fe}$ I $\mathrm{K} \alpha$ and Fe XXv He $\alpha$ lines. The GCXE spectrum cannot be explained the point source model, either (Figure 6a). There are large excesses at the three iron lines. Therefore, although the point-source 
scenario can well explain the GBXE, it can explain neither the ridge nor center. This result indicates unknown origins remaining in the GDXE.

For the GRXE, there is a significant residual in the Fe I K $\alpha$ line. As described in sec. 3, the scale height of the $\mathrm{Fe}$ I $\mathrm{K} \alpha$ line is similar to molecular clouds. A possible idea of the origin of the $\mathrm{Fe}$ I $\mathrm{K} \alpha$ line is local bombardment of low-energy cosmic-ray particles to $\mathrm{Fe}$ atoms in dense interstellar medium, such as molecular clouds. Since the cross section of the interaction has a peak at $\mathrm{MeV}$ energy, the $\mathrm{Fe}$ I $\mathrm{K} \alpha$ line would be a unique tracer of $\mathrm{MeV}$ protons.

For the GCXE, the three iron lines cannot be explained, and therefore additional components are required. They should have symbiotic phenomena of cold gas and very high temperature plasmas. The scale heights of the iron lines are $\sim 30 \mathrm{pc}$, which is similar to that of the Central Molecular Zone (CMZ), an active region where many stars and molecular gases are concentrating. Excess components are possibly related to big outbursts of $\mathrm{Sgr} \mathrm{A}^{*}$ in the past.

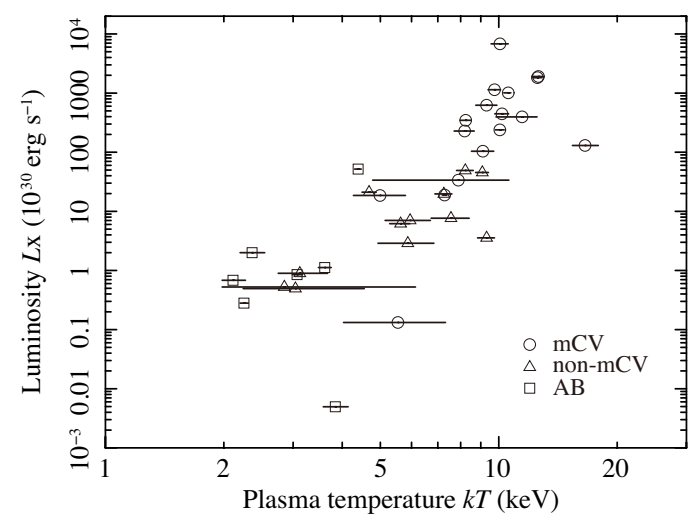

Figure 4: Plasma temperature $k T$ and luminosity $L_{\mathrm{X}}$ of $\mathrm{X}$-ray active stars used in this work. Those are divided into $\mathrm{mCV}$ (circle), non-mCV (triangle), and $\mathrm{AB}$ (square).

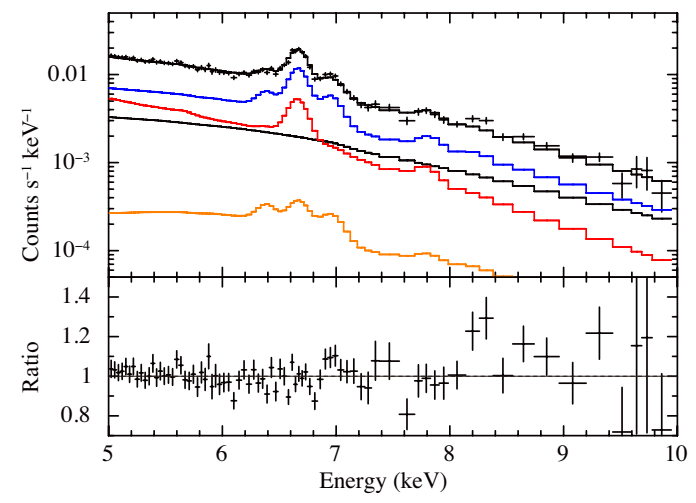

Figure 5: Upper panel: X-ray spectrum of "Bulge" fitted with a AS-combined model consisting of mCV (orange), non-mCV (blue), and $\mathrm{AB}$ (red) in addition to CXB (black). Lower panel: Ratio of the data to the model.

\section{Discussion at the meeting}

Based on the following comments given at the meeting, we discuss in this section. 



Figure 6: Same as figure 5 but for (a) "Center" and (b) "Ridge".

\section{DISCUSSION}

MARIO MACRI: Can you comment on the contribution of antimatter search in space experiments to the understanding of cosmological evolution?

Line-like residuals seen in the GDXE spectra (figures 5 and 6) might be of some emission line due to interaction of antimatters, for example, annihilation of matter and antimatter. However, no observational evidence has been found so far.

FRANCO GIOVANNELLI: The detection of exotic cosmic rays due to pair annihilation of dark matter particles in the Milky Way halo is a viable techniques to search for super-symmetric dark matter candidates. The study of the spectrum of gamma-rays, antiprotons and positrons offers good possibilities to perform this search in a significant portion of the Minimal Supersymmetric Standard Model parameter space.

Recently, some researches claimed detection of emission lines due to annihilation of sterile neutrinos near the Galactic center region [17, 18, 19, 20]. Since significance of the detection is not large, further observations are encouraged to constrain physical parameters of dark matter particles.

\section{References}

[1] Warwick, R. S., Turner, M. J. L., Watson, M. G., \& Willingale, R. The Galactic Ridge Observed by Exosat, Nature, 317, 218-221, 1985

[2] Revnivtsev, M., Sazonov, S., Gilfanov, M., Churazov, E., \& Sunyaev, R. Origin of the Galactic ridge $X$-ray emission, $A \& A, \mathbf{4 5 2}, 169-178,2006$

[3] Revnivtsev, M., Sazonov, S., Churazov, E., Forman, W., Vikhlinin, A., \& Sunyaev, R. Discrete sources as the origin of the Galactic X-ray ridge emission, Nature, 458, 1142-1144, 2009

[4] Koyama, K., Ikeuchi, S., \& Tomisaka, K. A Possible Contribution of Supernova-Remnants to the Excess X-Ray-Emission From the Galactic Ridge, PASJ, 38, 503-509, 1986

[5] Koyama, K., Maeda, Y., Sonobe, T., Takeshima, T., Tanaka, Y., \& Yamauchi, S. ASCA View of Our Galactic Center: Remains of Past Activities in X-Rays?, PASJ, 48, 249-255, 1996 
[6] Revnivtsev, M., Molkov, S., \& Sazonov, S. Map of the Galaxy in the 6.7-keV emission line, MNRAS: Letters, 373, L11-L15, 2006

[7] Koyama, K., Tsunemi, H., Dotani, T., Bautz, M. W., Hayashida, K., Tsuru, T. G., et al. X-ray Imaging Spectrometer (XIS) on board Suzaku, PASJ, 59, S23-S33, 2007

[8] Uchiyama, H., Nobukawa, M., Tsuru, T. G., \& Koyama, K. K-Shell Line Distribution of Heavy Elements along the Galactic Plane Observed with Suzaku, PASJ, 65, article id. 19, 2013

[9] Yamauchi, S., Nobukawa, K. K., Nobukawa, M., Uchiyama, H., \& Koyama, K. Scale heights and equivalent widths of the iron $K$-shell lines in the Galactic diffuse X-ray emission, PASJ, 68, article id.59, 2016

[10] Nobukawa, M., Uchiyama, H., Nobukawa, K. K., Yamauchi, S., \& Koyama, K., Origin of the Galactic Diffuse X-Ray Emission: Iron K-shell Line Diagnostics, ApJ, 833, article id. 268, 2016

[11] Kushino, A., Ishisaki, Y., Morita, U., Yamasaki, N. Y., Ishida, M., Ohashi, T., \& Ueda, Y. Study of the $X$-ray background spectrum and its large-scale fluctuation with ASCA, PASJ, 54, 327-352, 2002

[12] Mathis, J. S. in Allen's Astrophysical Quantities, 4th ed., ed. A. N. Cox (New York: Springer), ch. 21, 2000

[13] Stark, A. A., \& Lee, Y The Scale Height of Giant Molecular Clouds Is Less than That of Smaller Clouds, ApJ, 619, L159-L162, 2005

[14] Nobukawa, K. K., Nobukawa, M., Uchiyama, H., et al., Enhancement of the 6.4 keV Line in the Inner Galactic Ridge: Proton-induced Fluorescence?, ApJL $\mathbf{8 0 7}$ article id. L10, 2015

[15] Xu, X.-J., Wang, Q. D., \& Li, X.-D. Fe Line Diagnostics of Cataclysmic Variables and Galactic Ridge $X$-Ray Emission, ApJ, 818, article id. 136, 2016

[16] Warwick, R. S., Byckling, K., \& Pérez-Ramírez, D. The Galactic plane at faint X-ray fluxes - II. Stacked X-ray spectra of a sample of serendipitous XMM-Newton sources, MNRAS 438, 2967-2979, 2014

[17] Prokhorov, D., \& Silk, J. Can the Excess in the Fe XXVI Ly $\gamma$ Line from the Galactic Center Provide Evidence for $17 \mathrm{keV}$ Sterile Neutrinos? ApJL 725, L131, 2010

[18] Chan, M. H., \& Chu, M.-C. Observational Evidences for the Existence of 17.4 keV Decaying Degenerate Sterile Neutrinos Near the Galactic Center ApJL, 727, L47, 2011

[19] Riemer-Sørensen, S. Constraints on the presence of a $3.5 \mathrm{keV}$ dark matter emission line from Chandra observations of the Galactic centre A\&A, 590, A71, 2016

[20] Koyama, K., Kataoka, J., Nobukawa, M., et al., ASTRO-H White Paper - Plasma Diagnostic and Dynamics of the Galactic Center Region astro-ph, 1412.1170, 2014 